\title{
Numerical simulations of the interaction of accretion disks with young star magnetospheres
}

\author{
T. G. Yelenina ${ }^{1}$, G. V. Ustyugova ${ }^{1}$, and A. V. Koldoba ${ }^{2}$ \\ 1 Keldysh Institute for Applied Mathematics (KIAM), Russian Academy of Sciences, Miusskaya Square 4, 125047 Moscow, Russia \\ e-mail: elen@keldysh.ru; ustyugg@rambler.ru \\ 2 Institute of Mathematical Modelling (IMM), Russian Academy of Sciences, Miusskaya Square 4a, 125047 Moscow, Russia \\ e-mail: koldoba@spp.keldysh.ru
}

Received 30 March 2006 / Accepted 1 July 2006

ABSTRACT

\begin{abstract}
Aims. We present the results of the numerical simulations of the interaction between a magnetized star and an imperfectly conducting accretion disk. The star is rotating with constant angular velocity. The differentially rotating Keplerian disk is treated as a boundary condition. We are interested in the magnetic field topology dependence on the electrical conductivity of the disk.

Methods. To analyze the "star-disk" interaction we numerically investigate the MHD equations using Godunov-type high resolution numerical methods.

Results. It was found that in our model the "star-disk" interaction occurs with a quasi-periodic reconnection of the magnetic field coronal loops and plasmoid ejections. In the case of the perfect disk conductivity, the evolution of the coronal magnetic field leads to the periodic outflow of angular momentum from the disk. In the case of an imperfectly conducting disk, the configuration of the magnetic field is formed such that the disk angular momentum carried by the magnetic field is balanced by angular momentum carried by matter. It should be noted that we used the ideal MHD equation to obtain the solutions. The reconnection process in the disk corona depends on the numerical diffusivity that exists in our numerical code. Our simulations treat reconnection as occurring in current sheets. The thickness of the current sheet is broadened by numerical resistivity. Nevertheless, we suppose that the reconnection and plasmoid ejection takes place as well for real magnetic diffusivity. To verify the method and results we also used several more detailed grids to estimate the numerical diffusivity of the scheme. It is turned out that the setup model presented in the paper quite reasonable satisfies the goal of this paper, i.e., to investigate the regime of interaction between the magnetized star and the disk.
\end{abstract}

Key words. magnetohydrodynamics (MHD) - accretion, accretion disks - stars: magnetic fields - ISM: evolution methods: numerical

\section{Introduction}

This paper studies the evolution of the coronal magnetic field linked with a magnetized star and its accretion disk. We suggest that the plasma differential rotation along magnetic field lines is the reason of the "star-corona-disk" system evolution. We suppose that the magnetic field lines are frozen in the perfectly conducting coronal plasma. The differential rotation leads to the generation of the magnetic field toroidal component. Magnetic pressure increases in the inner part of the corona, and plasma is pushed towards the outer part together with the magnetic field lines. As a result there is the deformation or even the opening of poloidal magnetic field lines adopting a new configuration. The type of this new configuration is determined by several factors. One of them is the electrical conductivity of the relatively cold disk plasma.

We assume in this model that the imperfect plasma conductivity is essential only in the disk and is determined by velocity turbulent fluctuations. We consider the value of the turbulent magnetic diffusivity as a free parameter of the problem. To obtain an acceptable range for this parameter it is supposed that the coefficient of the turbulent magnetic diffusivity agrees in order of magnitude with the turbulent viscosity accepted in the standard $\alpha$-model of the Shakura-Sunyaev accretion disk (Shakura \& Sunyaev 1973).

We suppose the disk to be formed by relatively dense and cold matter. The disk is Keplerian, and the sound speed is much less than the Keplerian one, which means that the disk is geometrically thin. In this model, the disk is considered as an infinitely thin, conductive plane and as a boundary condition. It should be mentioned that the disk has a complicated structure and that its interaction with the magnetic field is not reduced to magnetic compression and magnetic field line slippage relative to matter (Balbus et al. 1995).

Besides the dynamics of the magnetic field, it is worth knowing its configuration after the opening of the magnetic field lines. This configuration defines the disk and the magnetic field evolution at large timescales. The main factor influencing this evolution is the rate of angular momentum transfer from the disk. The important role in this process also belongs to the magnetic field (Ferreira 1997).

A sufficiently strong magnetic field can also lead to the formation of a matter outflow from the disk to the corona. In case of a thin Keplerian disk, a criterion for the beginning of the "wind" formation has been given by Blandford \& Payne (1982). To generate this outflow from a Keplerian disk, the magnetic field line should be inclined to the rotation axis at an angle greater 
than $30^{\circ}$. In that case, a magnetic field line plays the role of a "rail" along which matter leaves the disk. Thus, the final magnetic field configuration strongly determines both the rate of disk accretion, through the rate of angular momentum outflow, and the rate of matter outflow along the magnetic field lines inclined to the rotation axis.

There are lots of papers concerning the evolution of the magnetic field interaction with a disk. We would like to mention some of them. Shu et al. (1994) presented steady state dynamics of accretion and outflow from a viscous and imperfectly conducting disk. Hayashi et al. (1996) presented resistive MHD simulations with a diffusive accretion disk and dipole stellar magnetic field topology. Ferreira (1997) gave a self-similar solution for the resistive disk for stationary MHD equations. Some papers presenting the results of ideal MHD simulations considered the disk as a boundary condition, including Ouyed \& Pudritz (1997) for dipole topology, Krasnopolsky et al. (1999), and Ustyugova et al. (1999) for an initially split-monopole magnetic field configuration in which they studied MHD outflows from the disk. Fendt \& Elstner $(1999,2000)$ studied the "star-disk" interaction and observed the opening of magnetic field lines and outflows. Kuwabara et al. (2000) introduced resistivity to simulate the effects of turbulent magnetic diffusivity. Romanova et al. (2002) studied the disk accretion on a rotating magnetized star with an aligned dipole moment and associated funnel flows; they described the "star-disk" interaction for the cases of quickly and slowly rotating stars. These simulations included a treatment of the vertical disk structure. Fendt \& Čemeljić (2002) studied the jet formation and propagation by used the resistive MHD equations. Uzdensky (2004) gave a review of a modern theoretical scenario of the "star-disk" system interaction, including both stationary and time-dependent approaches.

In this paper we consider the evolution of the magnetic field configuration from an initial dipole-like topology into the final one. We analyze the time-dependent ideal MHD equations using the high resolution Godunov-type method. We consider that the generated flow is axisymmetric and symmetric about the equatorial plane for a setup model of a "star-disk" system. The dependence of the disk angular momentum outflow rate on the disk surface electrical conductivity is investigated.

The structure of the paper is as follows. In the second section we propose the model and the evaluation of the disk surface magnetic diffusivity. Then we present the numerical method and describe our results for number disk surface magnetic diffusivity. Finally we formulate the main conclusions.

\section{Statement of the problem}

Let us consider a "star-corona-disk" system governed by the magnetic field (see Fig. 1). In our model the star has mass $M_{*}$, magnetic moment $\mu_{*}$, and angular velocity $\Omega_{*}$. It is assumed that the star rotation axis aligns with its magnetic axis $z$. The disk rotating around the rotating star is considered to be infinitely thin and located at $z=0$ for $R>R_{\mathrm{d}}$. It is supposed that the disk follows the Keplerian orbits around the star and that the disk is imperfectly conducting. The particle velocity in a Keplerian orbit with radius $r$ is $V_{\mathrm{k}}=\sqrt{G M_{*} / r}$, where $G=0.667 \times 10^{-7} \mathrm{~cm}^{3} \mathrm{~g}^{-1} \mathrm{~s}^{-2}$ is the gravitational constant. Thus, the disk is differentially rotating. In our model the star angular velocity $\Omega_{*}$ is large enough; therefore, the corotation radius $r_{\mathrm{c}}=\left(G M_{*} / \Omega_{*}^{2}\right)^{1 / 3}$ is less than the disk radius $R_{\mathrm{d}}$. If $r_{\mathrm{c}}>R_{\mathrm{d}}$, then matter flows to the star and the "funnel flow" regime probably takes place. However, we do not consider this case here.

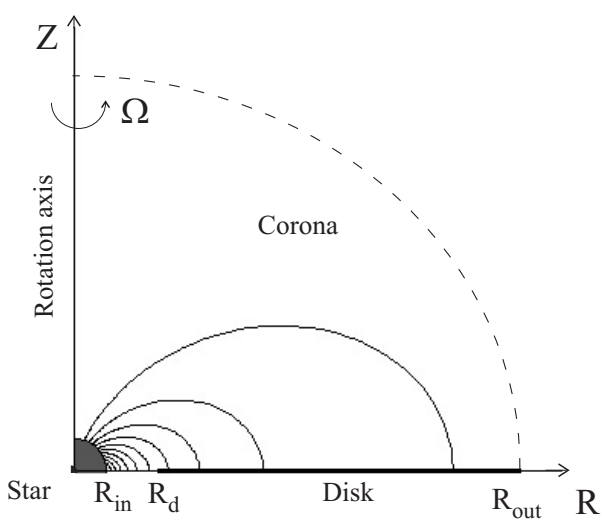

Fig. 1. Sketch of a magnetically linked "star-disk" system. The solid lines show the magnetic field lines. $R_{\text {in }}, R_{\text {out }}$ are the edges of the computational domain. The star is inside the computational domain, $R_{*}<R_{\text {in }}$.

The coronal plasma electro-conductivity is big enough that we can describe the flow by the system of the ideal MHD equations

$$
\begin{aligned}
& \frac{\partial \rho}{\partial t}+\nabla \cdot(\rho \boldsymbol{u})=0, \\
& \frac{\partial \rho \boldsymbol{u}}{\partial t}+\nabla \mathrm{T}=\rho \boldsymbol{g}, \\
& \frac{\partial \boldsymbol{B}}{\partial t}-\nabla \times(\boldsymbol{u} \times \boldsymbol{B})=0, \\
& \frac{\partial \rho S}{\partial t}+\nabla \cdot(\rho S \boldsymbol{u})=0, \\
& \nabla \boldsymbol{B}=0 .
\end{aligned}
$$

Here $\mathrm{T}_{i k}=\rho u_{i} u_{k}+p \delta_{i k}+\frac{1}{4 \pi}\left(-B_{i} B_{k}+\frac{B^{2}}{2} \delta_{i k}\right)$ is the stress tensor; $\boldsymbol{u}$ is the plasma velocity; $\boldsymbol{B}$ is the magnetic field; $\rho$ and $p$ are the plasma density and pressure; $S=p / \rho^{\gamma}$ is the entropy function; $\gamma$ is the adiabatic index; $\boldsymbol{g}=-\nabla \Phi_{\mathrm{g}}$ is the gravitational acceleration; $\Phi_{\mathrm{g}}=-G M_{*} / R$ is the star gravity potential; and $R$ is the distance from the gravitating center.

The system (1) is solved in spherical coordinates $(R, \varphi, \theta)$, with $\theta$ being the polar angle with the symmetry axis. Velocity $\boldsymbol{u}$ and magnetic field $\boldsymbol{B}$ have all their components $\boldsymbol{u}=(u, v, w)$ and $\boldsymbol{B}=\left(B_{R}, B_{\varphi}, B_{\theta}\right)$.

The aim of this paper is to investigate the character of the magnetic field evolution and field topology depending on the disk surface magnetic diffusivity $\zeta=c^{2} / 2 \pi \lambda$ ( $\lambda$ is surface electric conductivity, $c$ is the speed of light) at large time scales. As will be shown below, we used boundary conditions set on the equatorial plane $z=0$.

\subsection{Evaluation of the electric conductivity in the disk}

We suggest that turbulent diffusion of the magnetic field is determined by the same processes that determine turbulent viscosity, which leads to angular momentum transport in the disk. Thus, it is assumed that the value of turbulent magnetic diffusivity $\eta_{\mathrm{t}}$ is of the order of turbulent viscosity, like in the Shakura-Sunyaev model (Shakura \& Sunyaev 1973): $\eta_{\mathrm{t}}=\alpha_{\mathrm{t}} c_{\mathrm{s}} h$, where $c_{\mathrm{s}}$ is the sound speed in the disk, $h$ is the disk half-thickness, and $\alpha_{\mathrm{t}}$ is the dimensionless coefficient varying, according to Balbus (2003), in this range $0.01 \div 0.6$. 
Under hydrostatic equilibrium, the Keplerian disk half-thickness $h$ can be found from the relation: $(h / r)^{2}+b(h / r)-\left(c_{\mathrm{s}} / V_{\mathrm{k}}\right)^{2}=0$, where $b \equiv r\left(B_{r}^{2}+B_{\varphi}^{2}\right) /\left(4 \pi \Sigma V_{\mathrm{k}}^{2}\right)$, $\Sigma$ is the surface density, $V_{\mathrm{k}}$ is the Keplerian velocity, and $B_{r}$, $B_{\varphi}$ are the magnetic field components (Bisnovatyi-Kogan \& Lovelace 2001). In any case, even without taking the magnetic compression into account, the disk half-thickness satisfies the inequality $h \lesssim c_{\mathrm{s}} / \Omega_{k}$, where $\Omega_{\mathrm{k}}$ is the Keplerian angular velocity. Therefore, the turbulent magnetic diffusivity becomes $\eta_{\mathrm{t}} \lesssim \alpha_{\mathrm{t}} c_{\mathrm{s}}^{2} / \Omega_{\mathrm{k}}$.

The sound speed is much less than the Keplerian one due to the disk being cold. It means that $h \lesssim c_{\mathrm{s}} / \Omega_{\mathrm{k}} \ll r$, i.e., the disk is geometrically thin. In this model, the disk is considered to be an infinitely thin, conductive plane.

The turbulent electro-conductivity $\sigma_{\mathrm{t}}=c^{2} /\left(4 \pi \eta_{\mathrm{t}}\right)=$ $c^{2} /\left(4 \pi \alpha_{\mathrm{t}} c_{\mathrm{s}} h\right)$ is associated with the magnetic diffusivity $\eta_{\mathrm{t}}$. The surface disk conductivity is $\lambda=\int \sigma_{\mathrm{t}} \mathrm{d} z \sim 2 h \sigma_{\mathrm{t}}=c^{2} /\left(2 \pi \alpha_{\mathrm{t}} c_{\mathrm{s}}\right)$, and the surface magnetic diffusivity is

$\zeta=\frac{c^{2}}{2 \pi \lambda}=\alpha_{\mathrm{t}} c_{\mathrm{s}}=\alpha_{\mathrm{t}}\left(\frac{c_{\mathrm{s}}}{V_{\mathrm{k}}}\right) V_{\mathrm{k}}$.

In thin accretion disks, $c_{\mathrm{s}} / V_{\mathrm{k}}=h / r \ll 1$. Thus, an acceptable coefficient of the magnetic surface diffusivity is $\zeta=(0.01 \div$ $0.6)\left(c_{\mathrm{s}} / V_{\mathrm{k}}\right) V_{\mathrm{k}}$.

\subsection{Initial conditions}

We suggest that, at the initial time, the stellar magnetic field with dipole-like topology and magnetic moment $\mu_{*}$ penetrates the corona and the disk. So, the components of the magnetic field $\boldsymbol{B}$ are

$B_{R}=\frac{2 \mu_{*} \cos \theta}{R^{3}}, B_{\theta}=\frac{\mu_{*} \sin \theta}{R^{3}}, B_{\varphi}=0$.

We suppose that at the initial time $t=0$, the matter of the corona and the disk is in mechanical equilibrium with the force-free dipole magnetic field, i.e., the gravitational force is balanced with the "centrifugal" force (liquid particle acceleration) and pressure gradient.

The momentum equation for the system (1) is

$-\omega^{2} r \boldsymbol{e}_{r}+\frac{1}{\rho} \nabla p=-\nabla \Phi_{g}$,

where it is taken into account that the particles follow circular orbits. Here $\boldsymbol{e}_{r}$ is a cylindrical radius unit vector.

The magnetic pressure $\boldsymbol{B}^{2} / 8 \pi$ decreases as $1 / R^{6}$ due to the dipole-like field $\boldsymbol{B}$. Also, most of the simulation region is occupied by a plasma, where gas pressure dominates. This dense plasma prevents the opening of the magnetic field lines. It is not useful for our goals in this paper to address the case of the barotropic density distribution when $\rho=\rho(p)$ and angular velocity depends only on the cylindrical radius $r=R \sin \theta$. Let us consider that the density $\rho$ is a function not only of pressure $p$, but also of cylindrical radius $r: \rho=\rho(r, p)$. Let us denote $V(p, r)=1 / \rho$. The momentum equation along the $z$-axis (the projection of Eq. (2) on the $z$-axis) is

$V(p, r) \frac{\partial p}{\partial z}+\frac{\partial \Phi_{g}}{\partial z}=0$.

Integrating it by $z$ and assuming that $p \rightarrow 0$ under $z \rightarrow \infty$ and $\Phi_{g} \rightarrow 0$, we finally get

$\int_{0}^{p} V\left(p^{\prime}, r\right) \mathrm{d} p^{\prime}+\Phi_{g}=0$.
The integral is assumed to converge on its interior limit.

Let us consider now the momentum Eq. (2), along the radial direction

$-\omega^{2} r+V(p, r) \frac{\partial p}{\partial r}+\frac{\partial \Phi_{\mathrm{g}}}{\partial r}=0$.

After differentiating Eq. (3) by $r$ and subtracting Eq. (4) from it, we get

$\omega^{2} r+\int_{0}^{p} \frac{\partial V}{\partial r} \mathrm{~d} p^{\prime}=0$.

Thus, given the function $V(p, r)$, the relation (3) defines the function $p(r, z)$ and relation $(5)-$ the function $\omega(r, z)$.

If $V(p, r)=k(r) / p^{\alpha}, \alpha=$ const $<1$ (this condition is essential for the convergence of the integral in left part of Eq. (3), as $p \rightarrow 0$ ), then Eqs. (3) and (5) take the form

$\left\{\begin{array}{l}\frac{k p^{1-\alpha}}{1-\alpha}+\Phi_{g}=0, \\ \frac{p^{1-\alpha}}{1-\alpha} k^{\prime}+\omega^{2} r=0 .\end{array}\right.$

Integrating this set, we find the function $k(r)$

$\ln k=-\frac{1}{G M_{*}} \int \Omega^{2}(r) r^{2} \mathrm{~d} r$.

Here $\Omega(r)=\omega(r, 0)$ is the angular velocity at the equatorial plane $z=0$.

The angular velocity at the equatorial plane was chosen in the following way $\left(r_{1}, r_{2}\right.$ are the parameters of the problem and $\left.r_{1}=2 R_{\text {in }}, r_{2}=3 R_{\text {in }}\right)$ :

$\Omega(r)=\left\{\begin{array}{l}\Omega_{*}=\sqrt{G M_{*} / r_{1}^{3}}, 0<r<r_{1}, \\ \Omega_{*}+\frac{\sqrt{G M_{*} / r_{2}^{3}}-\Omega_{*}}{r_{2}-r_{1}}\left(r-r_{1}\right), \quad r_{1}<r<r_{2}, \\ \sqrt{G M_{*} / r^{3}}, r>r_{2} .\end{array}\right.$

As a result, the distributions of the pressure $p(r, z)$, density $\rho(r, z)$, and angular velocity $\omega(r, z)$ are obtained

$$
\begin{aligned}
& p(r, z)=\left(\frac{1-\alpha}{k(r)} \frac{G M_{*}}{\sqrt{r^{2}+z^{2}}}\right)^{1 /(1-\alpha)}, \rho(r, z)=\frac{p^{\alpha}(r, z)}{k(r)}, \\
& \omega=\sqrt{\frac{p^{1-\alpha}}{\alpha-1} \frac{k^{\prime}(r)}{r}}
\end{aligned}
$$

\subsection{Boundary conditions}

The surface currents in the disk (at $z=0$ ) lead to a discontinuity in the magnetic field disk-tangential components. Mathematically it means that the following condition is fulfilled (Landau \& Lifshitz 1982)

$n \times\left(B^{+}-B^{-}\right)=\frac{4 \pi}{c} i$

where $\boldsymbol{B}^{+}$and $\boldsymbol{B}^{-}$are the magnetic field under and over the disk respectively, $\boldsymbol{n}$ is the unit normal to the disk, and $\boldsymbol{n}$ is directed downward in cylindrical coordinates. Since we suggest that the MHD-flow is symmetric about the equatorial plane and consider the problem in the upper half-space, then $\boldsymbol{B}^{+}=-\boldsymbol{B}^{-}=\boldsymbol{B}$, and Eq. (8) gives

$\boldsymbol{n} \times \boldsymbol{B}+\frac{2 \pi}{c} \boldsymbol{i}=0$. 
Substituting the expression for the surface current in the disk $\boldsymbol{i}$, which due to Ohm's law at the comoving frame can be written as $\boldsymbol{i}=-\lambda(\boldsymbol{u}-\boldsymbol{V}) \times \boldsymbol{B} / c$, into the Eq. (9) for the current, we finally get

$(\boldsymbol{u}-\boldsymbol{V}-\zeta \boldsymbol{n}) \times \boldsymbol{B}=0$.

Assuming that the angular velocity $\omega$ and $r, \varphi$-components of the electric field in the comoving frame, change negligibly in the $z$-direction on scales of the order of the disk thickness $2 h$, we obtain $\lambda=\int \sigma_{\mathrm{t}} \mathrm{d} z \sim 2 h \sigma_{\mathrm{t}}$ for the disk surface electric conductivity.

In spherical coordinates, the tangential components of Eq. (10) are

$\left(v-V_{\mathrm{k}}\right) B_{\theta}-(w-\zeta) B_{\varphi}=0$,

$u B_{\theta}-(w-\zeta) B_{R}=0$.

The relations (11), (expressing Ohm's law for surface current in the disk), give two boundary conditions in the equatorial plane at $\theta=\pi / 2(z=0)$.

The real disk has some vertical (in the $z$-direction) structure that is not considered here. It is essential that thermodynamic parameters of the disk plasma change in the vertical direction, turning smoothly to the corona ones. So, the boundary conditions can be set arbitrarily, based on some physically reasonable assumptions.

We assume that matter outflow from the disk does not change its interior structure, i.e., the disk has sufficiently large mass, energy, and angular momentum. We assume that the specific entropy of the matter flowing out from the disk does not vary with time

$\left.S\right|_{\theta=\pi / 2}=S_{\mathrm{d}}(R)$.

We do not fix the density and the matter flux, which are determined via MHD equation solutions.

We have not yet discussed the question of why the outflows are originated. One possibility is the mechanism of Blandford \& Paine, which describes the generation of outflow in a sufficiently strong magnetic field inclined to the rotation axis at an angle greater than $30^{\circ}$ (Blandford \& Payne 1982). Other mechanisms are possible, too. However, it is necessary to study the internal structure of the disk to describe this process correctly. We do not consider these processes here. Instead, we treat the disk as a conventional plane and suppose that matter flows from the disk to the corona at a low velocity. It could be expected that plasma leaves the disk at a velocity less than the slow magnetosonic one. Let us accept that the $z$-component of the velocity is a fraction $\alpha_{\mathrm{c}}$ of the cusp one in this direction. The cusp velocity is not greater than slow magnetosonic one and has the same direction. The condition (12), which guarantees that the plasma outflow does not exceed the slow magnetosonic velocity, gives the boundary condition for $\theta=\pi / 2(\boldsymbol{a}=\boldsymbol{B} / \sqrt{4 \pi \rho}$ is the Alfvén velocity, parameter $\left.\alpha_{\mathrm{c}}(R)<1\right)$ :

$w+\alpha_{\mathrm{c}}(R) \frac{c_{\mathrm{s}}\left|a_{\theta}\right|}{\sqrt{c_{\mathrm{s}}^{2}+a^{2}}}=0$.

The condition (12) also guarantees that from any point of the disk, five characteristics can be seen. We do not study the dependence of the solution on the parameter $\alpha_{\mathrm{c}}$.

To get the equation describing the evolution of the magnetic flux function on the disk (at $\theta=\pi / 2$ ), let us use the induction equation

$\frac{\partial B_{\theta}}{\partial t}+\frac{c}{R} \frac{\partial R E_{\varphi}}{\partial R}=0$
Taking into account that on the disk $B_{\theta}=\partial \Psi /(R \partial R)$ and Ohm's law is fulfilled, the azimuthal component of the surface current, $E_{\varphi}+c B_{R} /\left(2 \pi \sigma_{t}\right)=0$. Integrating Eq. (13), we find

$\frac{\partial \Psi}{\partial t}+\zeta R B_{R}=0$

Let us formulate the boundary conditions on the inner boundary of the simulation region under $R=R_{\mathrm{in}}$. As for the boundary conditions at the equatorial plane, they are set for reasons of physical rationality. The main factor to take into account is on the one hand to arbitrarily choose the position of the inner boundary, i.e., the value of the inner radius of the simulation region $R_{\text {in }}$, and, on the other hand, to quickly (like $R^{-6}$ ) increase the magnetic pressure of the stellar dipole-like field. $R_{\text {in }}$ is chosen to be such that in some neighborhood of the inner boundary the star magnetic field has dominant influence on the plasma dynamics. In other words, in this region Alfvén velocity is much greater than both the gas sound speed and the Keplerian one. On the other hand, the choice of a very small $R_{\text {in }}$ is not reasonable from the computational point of view because in this case, the simulation region includes parts of the magnetosphere, where the Alfvén velocity is too large. It leads to an essential decreasing of the time integration step. Since in the model considered it is assumed that the magnetic field lines are frozen into the star surface rotating with angular velocity $\Omega_{*}$, on the inner boundary of the simulation region (and under it, right on the star surface) plasma moves along the rotating magnetic field lines. In the frame of the rotating star (on the inner boundary), the plasma velocity vector is parallel to the magnetic field one. Since transformation to the rotating frame does not change $\boldsymbol{B}$, but transforms $\boldsymbol{u}$ into $\boldsymbol{u}-V_{*} \boldsymbol{e}_{\varphi}$, where $V_{*}=\Omega_{*} R_{\text {in }} \sin \theta$, this boundary condition can be written in the following way $\left(R=R_{\text {in }}\right)$ :

$\left(\boldsymbol{u}-V_{*} \boldsymbol{e}_{\varphi}\right) \times \boldsymbol{B}=0$.

Condition (14) also implies that in the rotating frame, the electric field in the inner boundary is zero. This condition gives two boundary conditions under $R=R_{\text {in }}$.

On the outer boundary, at $R=R_{\text {out }}$, "free" boundary conditions are set. Such conditions should not influence the solution inside the simulation region preventing poloidal magnetic field lines from opening.

On the rotation axis, although it is not a boundary, the symmetry of the flow conditions for this axis are set:

$v=w=0, B_{\varphi}=B_{\theta}=0$, and $\Psi=0$, under $\theta=0$.

\section{Numerical method and results}

\subsection{Dimensionless variables and typical quantities for $T$ Tauri stars}

The dimensionless form of Eq. (1) is obtained in a standard way. As distance scale, $R_{0}$, we take one third of the distance from the star center to the inner edge of the disk. Thus, in dimensionless units the disk inner edge is $R_{\mathrm{d}}=3 R_{\mathrm{in}}$. The inner radius of the computational domain in dimensionless units is $R_{\text {in }}=1$. The time and the velocity scales are chosen so that in dimensionless units $G M_{*}=1$. This requirement yields $t_{0}=\sqrt{R_{0}^{3} / G M_{*}}$ as the time scale and $v_{0}=R_{0} / t_{0}$ as the velocity scale. As a magnetic field scale, $B_{0}$ is taken and then density, pressure, and magnetic moment scales are $\rho_{0}=B_{0}^{2} / v_{0}^{2}, p_{0}=B_{0}^{2}$, and $\mu_{* 0}=B_{0} R_{0}^{3}$.

As typical quantities of $\mathrm{T}$ Tauri stars, we adopt standard values like a star mass $M_{*}=0.8 M_{\odot}=1.6 \times 10^{33} \mathrm{~g}$ and 


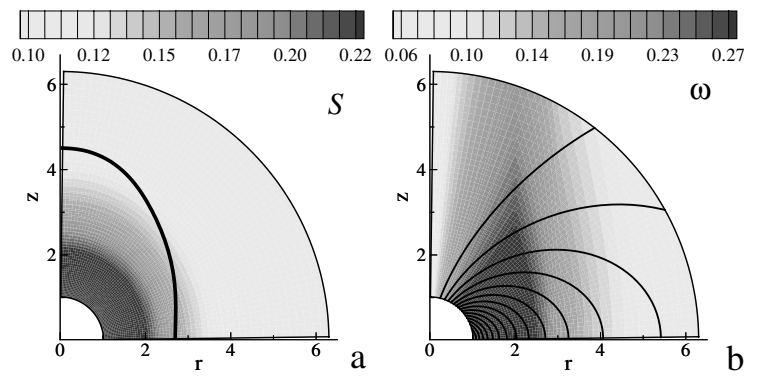

Fig. 2. a) Background colour shows the initial distribution of the specific entropy $S(r, z)$; the solid line presents the magnetic parameter $\beta=8 \pi p / B^{2}=1$. b) Background colour shows the initial distribution of the angular velocity $\omega(r, z)$; the thin lines present the magnetic flux function $\Psi(r, z)$.

$R_{\mathrm{d}}=9 R_{\odot}=5.4 \times 10^{11} \mathrm{~cm}$. Therefore, the distance scale is $R_{0}=R_{\mathrm{d}} / 3=1.8 \times 10^{11} \mathrm{~cm}$, and the time and velocity scales are $0.74 \times 10^{4} \mathrm{~s}$ and $2.43 \times 10^{7} \mathrm{~cm} \mathrm{~s}^{-1}$, respectively. The Keplerian rotation period at the disk inner edge is 8.3 days. The simulation region size is $1.134 \times 10^{12} \mathrm{~cm}$.

The star magnetic moment is taken such that the magnetic field on the star surface is $300 \mathrm{G}$, so $B_{0}=6.5 \mathrm{G}$. Thus, on the disk inner part, the dipole magnetic field is $2.4 \mathrm{G}, R_{*}=3 / 5 R_{\text {in }}$, $\mu_{*}=10$, and the magnetic moment is $3.8 \times 10^{35} \mathrm{G} \mathrm{cm}^{3}$. The density scale is $1.44 \times 10^{-14} \mathrm{~g} \mathrm{~cm}^{-3}$. We should note that in our calculations the plasma density in the disk corona is approximately 0.1 , which corresponds to $1.28 \times 10^{-15} \mathrm{~g} \mathrm{~cm}^{-3}$.

For the numerical integration of the ideal MHD Eq. (1), we use a Godunov-type conservative high resolution scheme (Kulikovskii et al. 1999; Yelenina \& Ustyugova 2004). To guarantee a divergence-free magnetic field, we apply the same procedure as Tóth (2000).

The system of Eq. (1) is integrated numerically in the region $R_{\text {in }}<R<R_{\text {out }}, 0<\theta<\pi / 2$. We use a non-uniform grid in the radial direction and a uniform one in the polar angle: $N_{\theta}=60$, $N_{R}=60$. The time step of the integration $\tau$ is restricted by the Courant condition.

To verify the method and results we also used two grids $(120 \times 60$ and $240 \times 120)$ and a first order numerical method to estimate the numerical diffusivity of the scheme. To satisfy the goals of this paper, i.e., to investigate the regime of interaction between the magnetized star and the disk, it is determined that the grid size presented in the paper is large enough.

For mathematical simulation of the interaction between a magnetized star and its accretion disk we performed several runs for different surface magnetic diffusivity $\zeta$. Figures $2-7$ present the results for the following values of parameter $\zeta: 0$, $0.001,0.005$. Figure 2 shows the initial system configuration at time moment, $t=0$.

The evolution of the coronal magnetic field loops in the "star-disk" system depends on the surface magnetic diffusivity $\zeta$. One can pick out the characteristic features that are essential for this process. For all the cases, poloidal field lines are pulled out and reconnected periodically (approximately each tenth rotation period of the disk's inner boundary). Then, a magnetic field toroidal component is generated. After reconnection, a plasmoid is formed. It is surrounded by the closed poloidal magnetic field lines along which the poloidal electric current runs. A plasmoid is determined by a strong toroidal magnetic field and a low gas pressure. The angular velocity inside the plasmoid is different from the corona one. $t=50$ is chosen for presenting the simulation results (the time is measured in the disk's inner boundary rotation period). Up to that moment, several reconnections of magnetic field lines took place, and next ejected plasmoid moves outwards to the outer boundary. Previous series of reconnections already led to the opening of the field lines close to the rotation axis. We should note that the reconnection of the magnetic field lines originates due to the numerical magnetic diffusivity. Nevertheless, we suppose that the reconnection and plasmoid ejection take place as well for real magnetic diffusivity.

Figures $3 \mathrm{a}-\mathrm{f}$ show the distributions of some variables for a magnetic diffusivity $\zeta=0$. It is clear from these pictures that the initial configuration has essentially changed and now it is not dipole-like. In the disk differential rotation region $(r>2)$, field lines come out of its surface with large slope angles (more than $30^{\circ}$ ), fulfilling the conditions for matter outflow from the disk. A toroidal magnetic field is generated as a result of the differential rotation, implying that there is a poloidal current in the corona, forming a double current sheet (background dark colour area in Fig. 3a). In the neighborhood of the star poloidal current, lines approximately coincide with the poloidal field ones. It is clear (see Fig. 3b) that the matter flows into the plasmoid area from the star and the disk's inner part. The plasmoid has hot matter surrounding it from the corona and moves up to the outer boundary. It is evident from comparing Figs. $3 \mathrm{a}$ and $3 \mathrm{c}$ that the angular velocity is practically constant along the field lines, especially near the star. It means that in this area the generation of the toroidal magnetic field does not take place, i.e., there are no poloidal electric currents. The magnetosphere, rotating with constant angular velocity, gives angular momentum to the plasmoid, twisting the matter inside it.

Figures $3 \mathrm{~d}-\mathrm{f}$ show the distributions of several quantities that describe the angular momentum transport in the system. The angular momentum conservation equation can be found from the continuity equation

$$
\frac{\partial \rho l}{\partial t}+\operatorname{div} \boldsymbol{L}=0
$$

where $\rho l=\rho u R \sin \theta$ is the angular momentum density. The poloidal components of the angular momentum flux density are

$$
\boldsymbol{L}=R \sin \theta\left(\rho v \boldsymbol{u}_{\mathrm{p}}-\frac{B_{\varphi} \boldsymbol{B}_{\mathrm{p}}}{4 \pi}\right),
$$

where $\boldsymbol{u}_{\mathrm{p}}, \boldsymbol{B}_{\mathrm{p}}$ are the plasma poloidal velocity and the poloidal magnetic field. The first term in the right part describes the angular momentum transported by matter, the second one the angular momentum by magnetic field.

There are two areas of intense momentum transport (see Fig. 3d). One of them is near the star pole where transport takes place due to magnetic stresses. The second one is above the disk, in the area of its differential rotation where the transport is caused by the matter flow. These processes are presented in Figs. $3 \mathrm{e}$ and $\mathrm{f}$ in more detail. It is clear from Fig. $3 \mathrm{f}$ that the angular momentum from the star is mainly transported by magnetic field. Also, there is intense momentum transport inside the plasmoid.

The distributions for the same variables at $t=50$ are shown in Figs. 4 and 5 in case of a finite surface magnetic diffusivity. Figures $4 \mathrm{a}-\mathrm{f}$ correspond to $\zeta=0.001$, Figs. $5 \mathrm{a}-\mathrm{f}$ to $\zeta=0.005$.

Figures $6 \mathrm{a}-\mathrm{c}$ show the influence of the magnetic diffusivity on the magnetic field topology. Level lines of the magnetic flux function $\Psi(r, z)$ for all cases (a: $\zeta=0, \mathrm{~b}: \zeta=0.001, \mathrm{c}: \zeta=0.005)$ are chosen at $t=11$. At this time, the first reconnection took place for the case $\zeta=0$. It is clear (see Figs. 6a-c) that the less the disk conductivity (i.e., the more magnetic diffusivity), the 

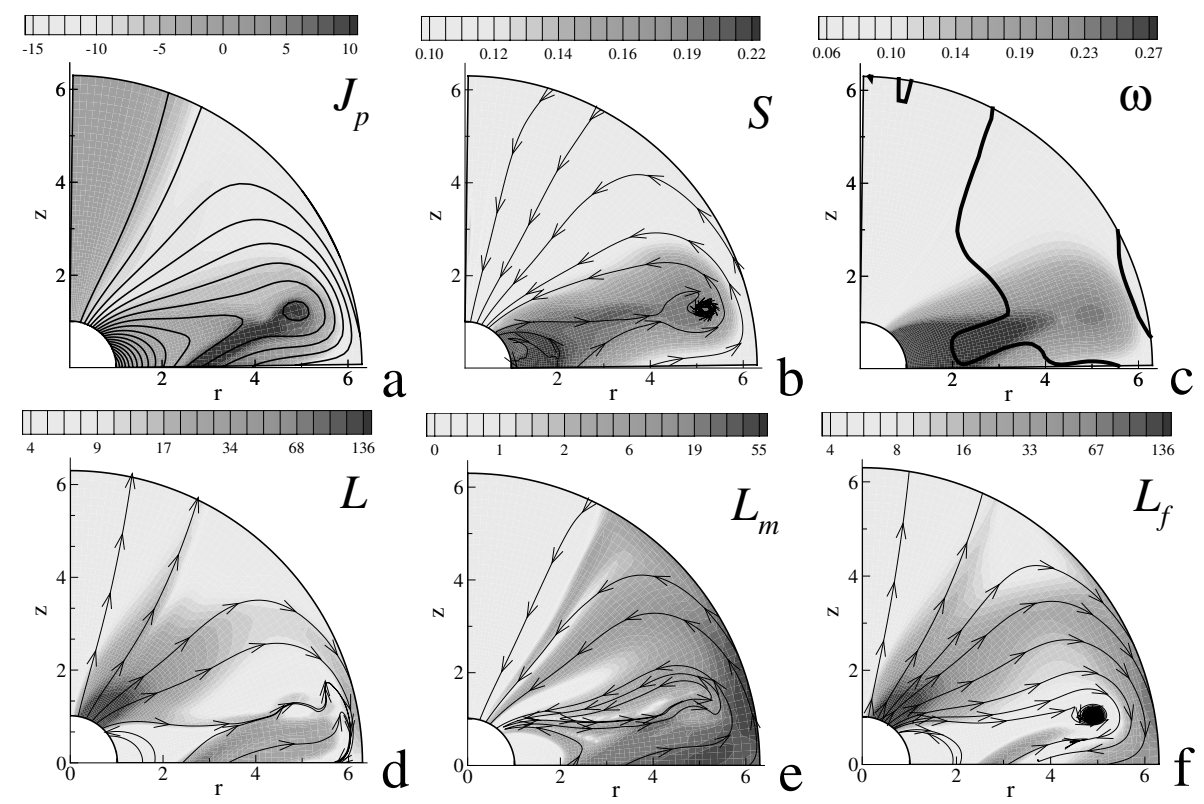

Fig. 3. Flow distributions for $\zeta=0.0$ at $t=50$. a) Background colour shows the poloidal current $J_{\mathrm{p}}=R \sin \theta B_{\varphi}$. The magnetic field lines are shown by solid lines. There is a poloidal current in the corona, forming a double current sheet (shown by background dark colour region). b) Background colour shows the specific entropy $S$. Streamlines present the matter flux. The plasmoid has hot matter surrounding it from the corona and moves up to the outer boundary. c) Background colour shows the plasma angular velocity $\omega$; the solid lines present the level of the plasma parameter $\beta=1$. In the neighborhood of the star poloidal current, lines approximately coincide with the poloidal field ones. Figures d)-f) show the distribution of the angular moment transport in the system. d) Background colour shows the magnitude of the angular momentum flux, and streamlines show the direction of the system angular momentum transport. e) Streamlines show the angular momentum flux direction carried by matter. The background colour shows the magnitude of this vector. f) Streamlines present the angular momentum flux direction, carried by the magnetic field, and its magnitude is shown by colour. There are two regions of intense momentum transport. One of them is near the star pole, where transport takes place due to magnetic stresses f). The second one is above the disk, in the area of its differential rotation, where the transport is caused by matter flow e).

more the distance from the axis and the disk where the plasmoid ejection takes place. In case of imperfect disk conductivity, field lines are no longer frozen into the disk and, twisted by the star, they are shifted outwards. We can say that the more the surface magnetic viscosity $\zeta$, the slower the evolution takes place, and the more seldom plasmoids are formed in the corona.

The matter flowing out from the disk and the magnetic field both take away the angular momentum from the disk. The whole angular momentum flux taken away from the disk per unit time is

$L=L_{\mathrm{m}}+L_{\mathrm{f}}=-\int \rho(\boldsymbol{r} \times \boldsymbol{u}) \boldsymbol{u} \mathrm{d} \boldsymbol{S}+\frac{1}{4 \pi} \int(\boldsymbol{r} \times \boldsymbol{B}) \boldsymbol{B} \mathrm{d} \boldsymbol{S}$.

Here, the integration is over the disk surface, and $\mathrm{d} \boldsymbol{S}$ is the element of disk surface directed outwards (from the simulation region). The first term $L_{\mathrm{m}}$ is the angular momentum flux carried by matter; the second one $L_{\mathrm{f}}$ is the angular momentum flux carried by magnetic field.

The time dependence of the angular momentum fluxes carried by matter $L_{\mathrm{m}}$, by magnetic field $L_{\mathrm{f}}$, and by their sum $L=L_{\mathrm{m}}+L_{\mathrm{f}}$ is shown in Figs. 7a-c for different values of $\zeta$.

For $\zeta=0$ (see Fig. 7a), the process of angular momentum transport from the disk to the corona is quasi-periodic, with one period approximately equal to ten rotation periods of the disk's inner part. After $t_{*}=45$, there is a relaxation of the system accompanied by small oscillations of the angular momentum fluxes. During this time, no new plasmoids are generated. After $t=65$, the reconnection process of the magnetic field lines is resumed. It can be seen from the angular momentum fluxes oscillations (see Fig. 7a).

The finite disk conductivity changes the course of events (interaction between magnetic star and accretion disk). As it is seen from Figs. 7a-c, the magnetic lines reconnection lasts until some time $t_{*}$ (depending on $\zeta$ ). The more the magnetic diffusivity $\zeta$, the later the plasmoids ejection begins and the earlier it ends. We should note that in case of an imperfect disk conductivity, the coronal magnetic field evolution is qualitatively similar to the perfect one. The reconnection of the magnetic field lines takes place at the time corresponding to the maximum angular momentum outflow carried by the magnetic field from the disk. In contrast to a perfect disk conductivity $(\zeta=0)$, the activity of the reconnection slows down and the system relaxes to such a state that no new plasmoids are generated. The disk angular momentum carried by the magnetic field is balanced by angular momentum carried by matter. Non-frozen in the disk, magnetic field lines move along the disk, which lead to the increasing of the magnetic flux in the disk. According to our simulations, matter moves at an appreciable velocity, and no new reconnection takes place if the disk surface conductivity is large enough.

\section{Summary and conclusions}

The influence of the disk surface magnetic diffusivity on the "star-corona-disk" system evolution was studied. For all cases, poloidal field lines are pulled out and are reconnected periodically, approximately each tenth rotation period of the disk's inner boundary. The magnetic field configuration has essentially changed from the dipole-like one. The magnetic field lines come out of its surface with a large slope angle, fulfilling the conditions for matter outflow from the disk, in the disk differential rotation region.

The more the magnetic diffusivity, the more the distance from the axis and the disk where the plasmoid ejection takes 

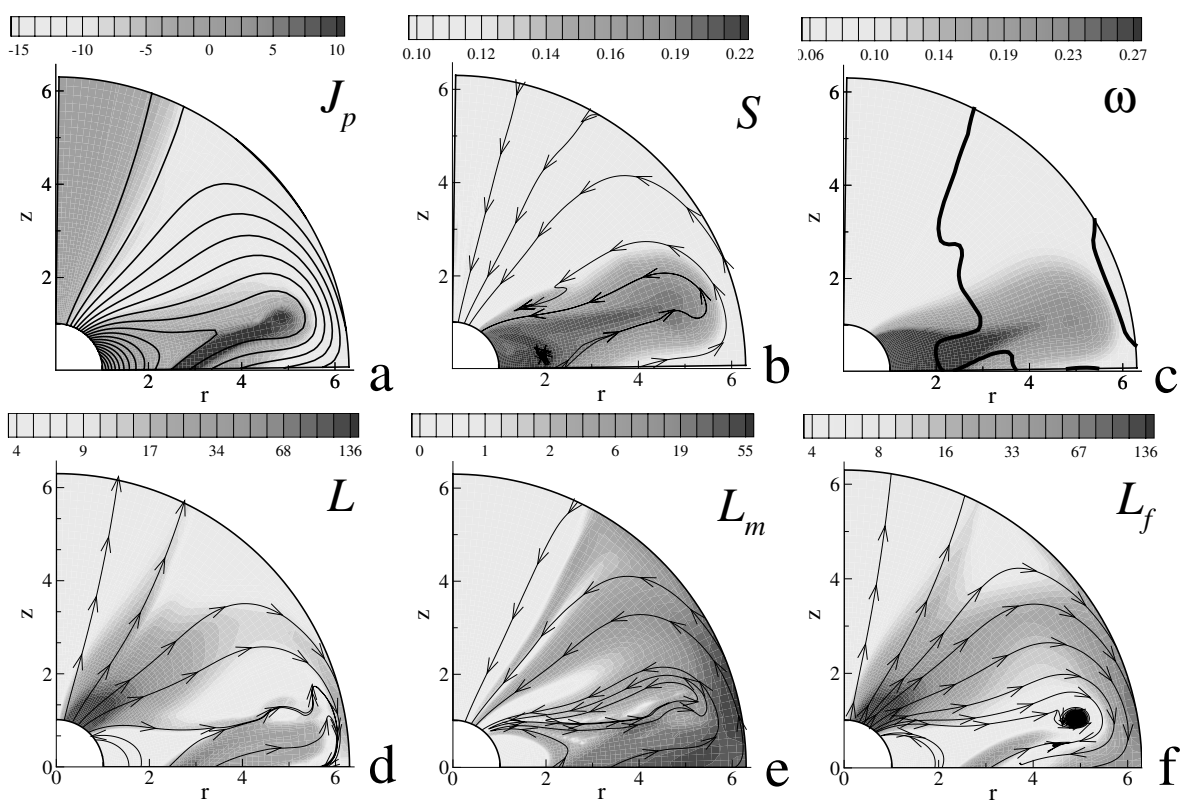

Fig. 4. Flow distributions for $\zeta=0.001$ at $t=50$. a) Background colour shows the poloidal current $J_{\mathrm{p}}$. The magnetic field lines are shown by solid lines. b) Background colour shows the specific entropy $S$. Streamlines present the matter flux. c) Background colour shows the plasma angular velocity $\omega$. A solid line presents the plasma parameter level $\beta=1$. Figures d)-f) show the distribution of the angular moment transport in the system. d) Background colour shows the magnitude of the angular momentum flux, and streamlines show the direction of the system angular momentum transport. e) Streamlines show the angular momentum flux direction carried by matter. The background colour shows the magnitude of this vector. f) Streamlines show the angular momentum flux direction, carried by the magnetic field; its magnitude is presented by colour.
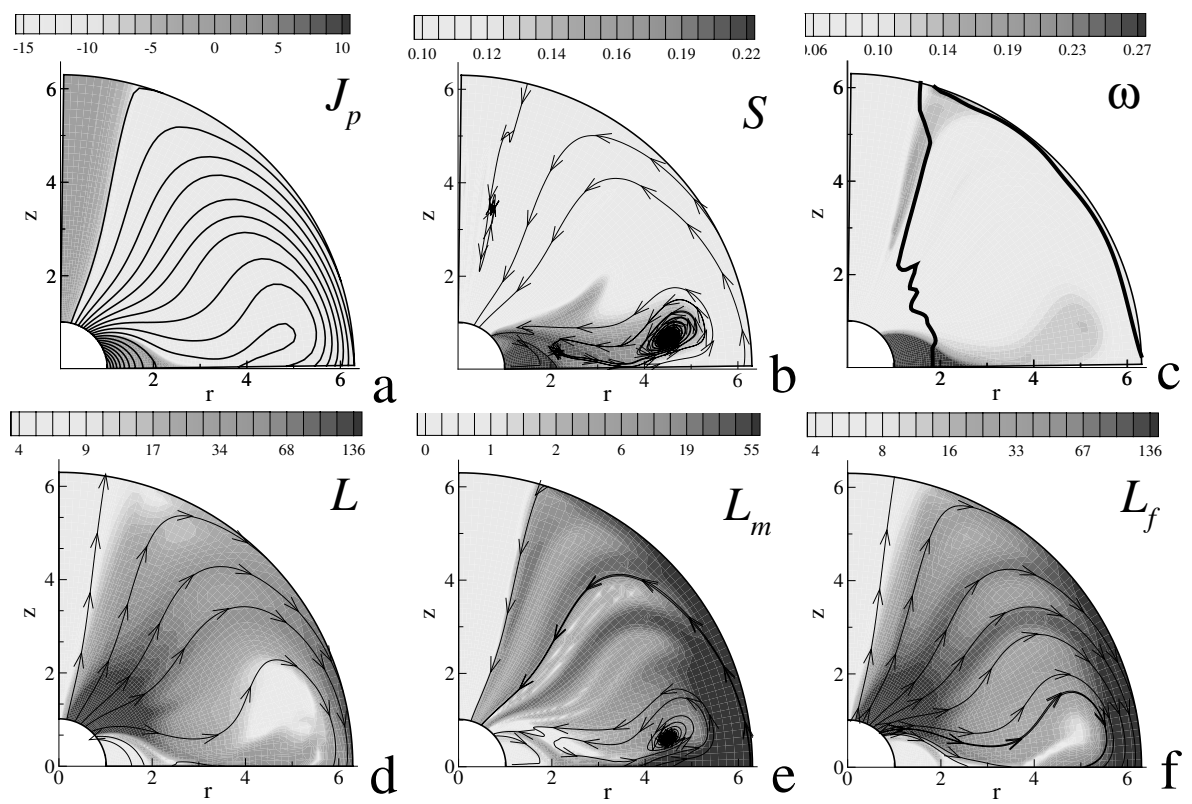

Fig. 5. Flow distributions for $\zeta=0.005$ at $t=50$. a) Background colour shows the poloidal current $J_{\mathrm{p}}$. The magnetic field lines are shown by solid lines. b) Background colour shows the specific entropy $S$. Streamlines present the matter flux. c) Background colour shows the plasma angular velocity $\omega$; the solid line presents the plasma parameter level $\beta=1$. Figures d) $-\mathbf{f}$ ) show the distribution of the angular moment transport in the system. d) Background colour shows the magnitude of the angular momentum flux, and streamlines show the direction of the system angular momentum transport. e) Streamlines show the angular momentum flux direction carried by matter. The background colour shows the magnitude of this vector. f) Streamlines show the angular momentum flux direction, carried by the magnetic field; its magnitude is presented by colour.

place. In the case of imperfect disk conductivity, magnetic field lines are no longer frozen into the disk and, twisted by the star, they are shifted outwards. The more the surface magnetic diffusivity $\zeta$, the slower the evolution takes place and more seldom plasmoids are formed in the corona.

In the case of imperfect disk conductivity, the coronal magnetic field evolution is qualitatively similar to the perfect one.
The reconnection of the magnetic field lines takes place at the time corresponding to the maximum angular momentum outflow carried by the magnetic field from the disk. In contrast with perfect disk conductivity, the activity of the reconnection slows down and the system relaxes to such a state that no new plasmoids are generated. The disk angular momentum carried by magnetic field is balanced by angular momentum carried by 

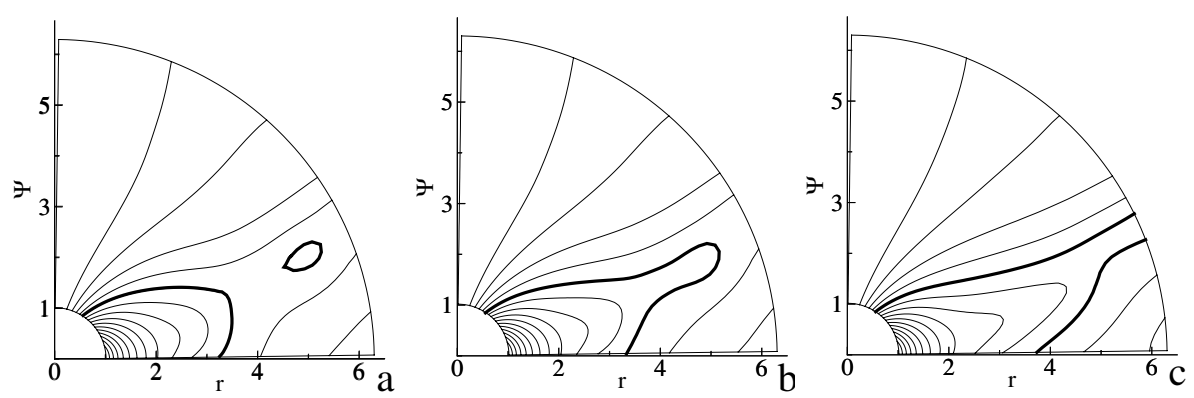

Fig. 6. a)-c) Influence of the surface magnetic diffusivity $\zeta$ on the magnetic field topology at $t=11$ when the first reconnection took place for the case $\zeta=0$. Level lines of the magnetic flux function $\Psi(r, z)$ are shown by thin lines (magnetic field lines: on a) for $\zeta=0.0$, on $\mathbf{b}$ ) for $\zeta=0.001$, and on c) for $\zeta=0.005$ ). $\Psi=151.6$ is shown by a solid line. The more the magnetic diffusivity, the more the distance from the axis and the disk where the plasmoid ejection takes place. In case of imperfect disk conductivity, field lines are no longer frozen into the disk and are shifted outwards.
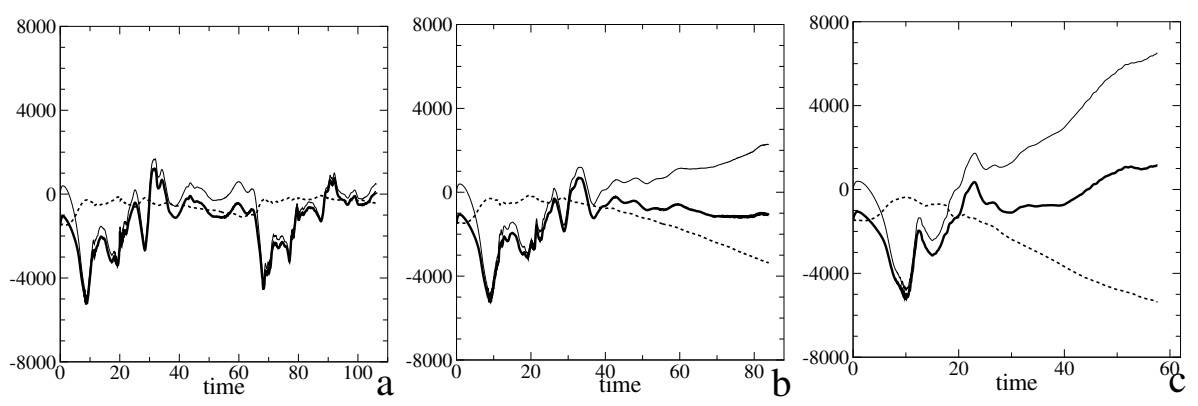

Fig. 7. Time dependence of the angular momentum fluxes carried by matter $L_{\mathrm{m}}$, by magnetic field $L_{\mathrm{f}}$, and total $L=L_{\mathrm{m}}+L_{\mathrm{f}}$ for different $\zeta$ : on a) for $\zeta=0.0$, on b) for $\zeta=0.001$, and on $\mathbf{c}$ ) for $\zeta=0.005 ; L_{\mathrm{m}}$ is shown by a dashed line, $L_{\mathrm{f}}$ is shown by a thin line, and $L$ is shown by a solid line. For $\zeta=0$ a), the process of angular momentum transport from the disk to the corona is quasi-periodic, with a period approximately equal to ten rotation periods of the disk's inner part. Then, the relaxation the process is resumed. For $\zeta=0.001 \mathbf{b}$ ) and $\zeta=0.005 \mathbf{c}$ ), the magnetic line' reconnection lasts for some time. The more the magnetic diffusivity $\zeta$, the earlier the plasmoids ejection ends. The reconnection of magnetic field lines takes place at the time corresponding to the maximum angular momentum outflow carried by the magnetic field from the disk. In contrast to a case for $\zeta=0$, the activity of the reconnection slows down and the system relaxes to such a state that no new plasmoids are generated. The disk angular momentum carried by the magnetic field is balanced by angular momentum carried by matter, in particular, $|L| \ll\left|L_{\mathrm{f}}\right|$.

matter. Magnetic field lines that are not frozen in the disk move along the disk and lead to the increasing of the magnetic flux in the disk.

It should be noted that we used the ideal MHD equation to obtain the solutions. The reconnection process in the corona and the plasmoid ejection depends on the numerical diffusivity that exists in our numerical code. Our simulations treat the reconnection as occurring in the current sheets. The thickness of the current sheet is broadened by the numerical resistivity. Nevertheless, we suppose that the reconnection and the plasmoid ejection take place as well for the real magnetic diffusivity.

Summing up, we can make following conclusions.

1. In the case of a perfectly conducting disk, the evolution of the coronal magnetic field leads to a quasi-periodic outflow of the angular momentum from the disk. The interaction process occurs with the reconnection of the magnetic field lines and plasmoid ejections.

2. In the case of an imperfectly conducting disk, the configuration of the magnetic field lines is formed such that the angular momentum flux carried by the magnetic field from the disk becomes balanced by the flux transported by matter.

Acknowledgements. We would like to thank Marina Romanova, Miljenko Čemeljić, Sergey Ustyugov and our referee Frank Shu for useful suggestions and helpful comments about the paper. Part of this work was supported by the Russian Foundation of Basic Research, RFBR project number 06-02-16608, the Scientific School Program project number 9399.2006.2, and the RAS Presidium Program number 14.

\section{References}

Balbus, S. A. 2003, ARA\&A, 41, 555

Bisnovatyi-Kogan, G. S., \& Lovelace, R. V. E. 2001, New Astron. Rev., 45, 663 Blandford, R. D., \& Payne, D. G. 1982, MNRAS, 199, 883

Fendt, C., \& Čemeljić, M. 2002, A\&A, 395, 1045

Fendt, C., \& Elstner, D. 1999, A\&A, 349, L61

Fendt, C., \& Elstner, D. 2000, A\&A, 363, 208

Ferreira, J. 1997, A\&A, 319, 340

Hawley, J. F., Gammie, C. F., \& Balbus, S. A. 1995, ApJ, 440, 742

Hayashi, M. R., Shibata, K., \& Matsumoto, R. 1996, ApJ, 468, L37

Krasnopolsky, R., Li, Z.-Y., \& Blandford, R. 1999, ApJ, 526, 631

Kulikovskii, A. G., Pogorelov, N. V., \& Semenov, A. Yu. 2001, Mathematical Aspects of Numerical Solution of Hyperbolic Systems (Boka Raton, FL; London: Chapman \& Hall/CRC)

Kuwabara, T., Shibata, K., Kudoh, T., \& Matsumoto, R. 2000, PASJ, 52, 1109

Landau, L. D., \& Lifshitz, E. M. 1982, Electrodynamics of Continuous Media (Moscow: Nauka)

Ouyed, R., \& Pudritz, R. E. 1997, ApJ, 482, 712

Romanova, M. M., Ustyugova, G. V., Koldoba, A. V., \& Lovelace, R. V. E. 2002, ApJ, 578, 420

Shakura, N. I., \& Sunyaev, R. A. 1973, A\&A, 24, 337

Shu, F., Najita, J., Ostriker, E., et al. 1994, ApJ, 429, 781

Tóth, G. 2000, J. Comput. Phys., 161, 605

Ustyugova, G. V., Koldoba, A. V., Romanova, M. M., Chechetkin, V. M., \& Lovelace, R. V. E. 1999, ApJ, 516, 221

Uzdensky, D. A. 2004, Ap\&SS, 292, 573

Yelenina, T. G., \& Ustyugova, G. V. 2004, Prepint KIAM RAS, No. 73 